\title{
Nonlinear Loading-Rate-Dependent Force Response of Individual Vimentin Intermediate Filaments to Applied Strain
}

\author{
Johanna Block, ${ }^{1}$ Hannes Witt, ${ }^{2}$ Andrea Candelli, ${ }^{3,4}$ Erwin J. G. Peterman, ${ }^{3}$ Gijs J. L. Wuite, ${ }^{3}$ \\ Andreas Janshoff, ${ }^{2}$ and Sarah Köster, ${ }^{1, *}$ \\ ${ }^{1}$ Institute for X-Ray Physics, Georg-August-Universität Göttingen, 37077 Göttingen, Germany \\ ${ }^{2}$ Institute of Physical Chemistry, Georg-August-Universität Göttingen, 37077 Göttingen, Germany \\ ${ }^{3}$ Department of Physics and Astronomy and LaserLab, Vrije Universiteit Amsterdam, 1081 HV Amsterdam, Netherlands \\ ${ }^{4}$ LUMICKS B.V., 1081 HV Amsterdam, Netherlands \\ (Received 13 September 2016; published 24 January 2017)
}

\begin{abstract}
The mechanical properties of eukaryotic cells are to a great extent determined by the cytoskeleton, a composite network of different filamentous proteins. Among these, intermediate filaments (IFs) are exceptional in their molecular architecture and mechanical properties. Here we directly record stress-strain curves of individual vimentin IFs using optical traps and atomic force microscopy. We find a strong loading rate dependence of the mechanical response, supporting the hypothesis that IFs could serve to protect eukaryotic cells from fast, large deformations. Our experimental results show different unfolding regimes, which we can quantitatively reproduce by an elastically coupled system of multiple two-state elements.
\end{abstract}

DOI: 10.1103/PhysRevLett.118.048101

The eukaryotic cytoskeleton is a crucial determinant of many cellular properties, including cell mechanics. Three types of filamentous proteins, microfilaments (MFs), microtubules (MTs), and intermediate filaments (IFs) interact with numerous cross-linking proteins and molecular motors, and the emerging composite network spans the whole cell [1]. Whereas MFs and MTs are conserved across tissues and organisms, IFs are expressed in a cell-type specific manner and 70 different IF coding genes have been found in humans [2]. Interestingly, IFs have recently been found to play a direct role in cell mechanics [3,4]. All cytoskeletal IFs share a tripartite monomer structure consisting of an $\alpha$-helical "rod" domain and intrinsically disordered "head" and "tail" regions [see Fig. 1(a), top]. The molecular assembly of IFs follows a strictly hierarchical path, including lateral assembly into dimers, tetramers, and unit length filaments (ULFs), followed by longitudinal annealing leading to $10 \mathrm{~nm}$ diameter filaments that can be many $\mu \mathrm{m}$ long, as shown schematically in Fig. 1(a), bottom.

IFs show interesting mechanical properties [2,8-10] such as high flexibility, with a persistence length of few $\mu \mathrm{m}$ $[11,12]$ and extreme extensibility $[13,14]$. Both properties originate from the open molecular architecture of the filaments. The extensibility of different IFs has previously been measured by lateral stretching of adsorbed IFs using atomic force microscopy (AFM) [13-15] and force-strain curves for vimentin dimers and tetramers have been

Published by the American Physical Society under the terms of the Creative Commons Attribution 3.0 License. Further distribution of this work must maintain attribution to the author(s) and the published article's title, journal citation, and DOI. modeled by molecular dynamics simulations [16]. The AFM experiments have revealed that IFs can be extended up to 3.6 times the original length before they rupture. However, it is in the nature of the measurement that the filaments may be imaged before and after stretching, but not in situ during the process. Since the filaments are stretched laterally and interact with the substrate during the extension a precise and quantitative assessment of the elastic properties could not be achieved.

Here, we directly probe the loading rate dependent mechanical properties of individual vimentin IFs under physiological buffer conditions using optical traps (OT) and AFM to stretch the filaments along their long axis up to a strain of 3.5. A strong loading rate dependency of the mechanical response is found and explained by an elastically coupled system of multiple two-state elements.

Human vimentin C328A with an additional cysteine at the $C$-terminus was recombinantly expressed in $E$. coli and purified from inclusion bodies [17]. Labeling with ATTO647N-maleimide (AttoTech GmbH, Siegen, Germany) or biotin-maleimide (Jena Bioscience $\mathrm{GmbH}$, Jena, Germany) was performed according to Ref. [18] with minor changes for the biotin labeling. The biotin-labeled vimentin and any remaining free biotin were separated by $2 \times$ elution over PD25 Miditrap columns (GE Healthcare Freiburg, Germany). In the first run, the column was equilibrated with a labeling buffer and eluted with a storage buffer (buffers according to Ref. [18]). In the second run the column was equilibrated and eluted with a storage buffer. The protein concentration was monitored by measuring the absorption at $280 \mathrm{~nm}$ (Nanodrop ND-1000, ThermoScientific Technologies, Inc.,Wilmington, USA). Labeled and unlabeled vimentin was stored at $-80^{\circ} \mathrm{C}$ 


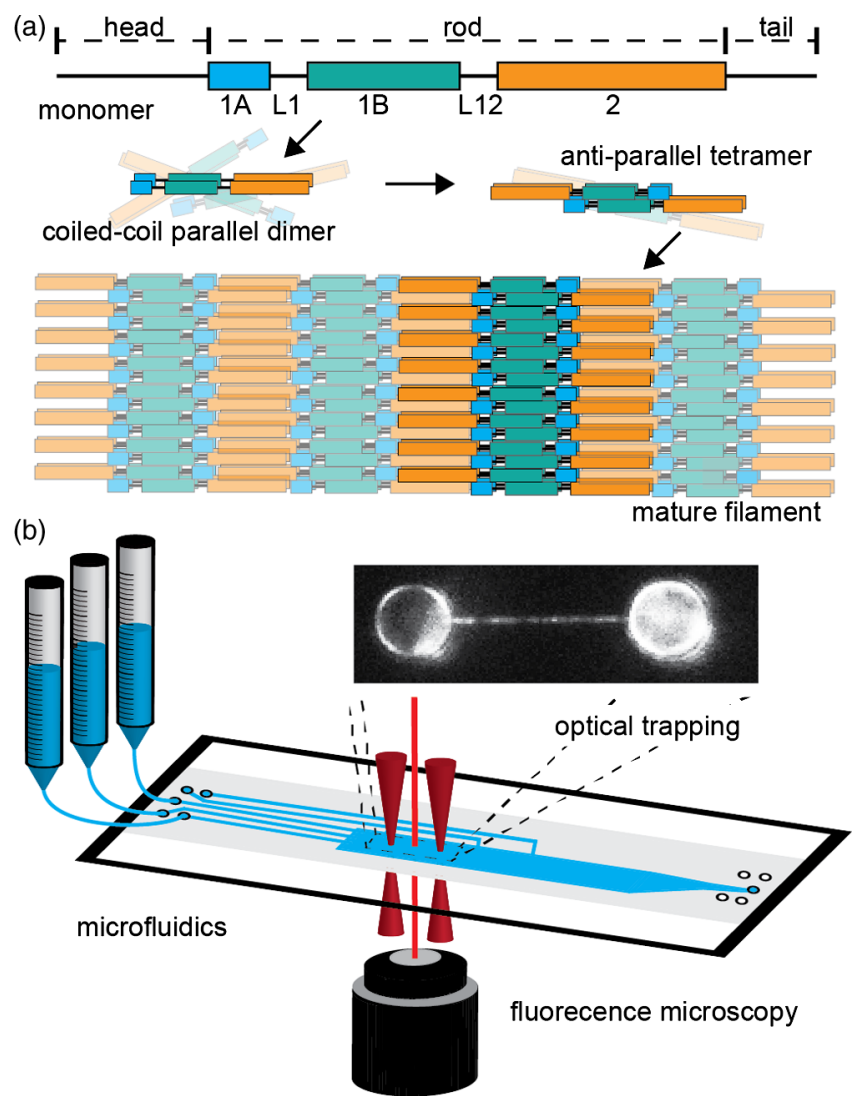

FIG. 1. (a) Schematic representation of vimentin filament formation. Vimentin monomers contain a head, a tail, and a central rod domain. The rod domain is composed of three $\alpha$-helical coils [1A: 36 amino acids (aa), 1B: 92 aa and 2: 139 aa] and two linkers (L1 and L12) [5-7]. Two monomers form a parallel coiled-coil dimer and two dimers form a half-staggered antiparallel tetramer. Via unit-length filaments (ULFs, composed of typically eight tetramers, not shown) mature filaments are formed by longitudinal annealing. (b) Overview of the experimental setup used for OT experiments, combining optical traps and confocal imaging with a microfluidic device. The inset shows a fluorescently labeled vimentin filament trapped between two $4.5-\mu \mathrm{m}$ beads.

$[12,18]$. Prior to assembly into filaments, the vimentin protein was dialyzed stepwise into a $2 \mathrm{mM}$ sodium phosphate buffer, $p H 7.5$ [12]. Assembly was performed at a vimentin concentration of $0.2 \mathrm{~g} / \mathrm{L}$ by dialysis into a $2 \mathrm{mM}$ sodium phosphate buffer, $p H$ 7.5, containing $100 \mathrm{mM} \mathrm{KCl}$ (assembly buffer), at $37{ }^{\circ} \mathrm{C}$ over night [19]. About $2 \%$ of the monomers were ATTO647N labeled and about $15 \%$ were biotin labeled. Before OT and AFM measurements, vimentin filaments were diluted 1:1000 and 1:10 with assembly buffer, respectively. OT measurements were performed with a setup combining optical traps, a confocal fluorescence microscope, and a microfluidic system (LUMICKS, Amsterdam, Netherlands) [20,21] [Fig. 1(b)]. Streptavidincoated polystyrene beads $(4.5 \mu \mathrm{m}$, Spherotech, Inc., Lake Forest, IL, USA), filaments, and buffer were flushed separately into the microfluidic channel by air pressure. Because of laminar flow, the three solutions did not mix in the channel part where the measurements were executed. Every measurement was started by capturing two beads with the OTs. By moving the microfluidic device with respect to the OTs, filaments were attached to the beads and moved into the buffer channel. Subsequently stopping the flow ensured that only one filament was attached between the two beads, as controlled by imaging (see movie in the Supplemental Material [22]). Filaments were stretched to a maximum force of about $800 \mathrm{pN}$ with different loading rates, varying from 0.05 to $4.5 \mu \mathrm{m} / \mathrm{s}$.

AFM experiments were performed using a commercial instrument (MFP-3D Bio, Asylum Research, Santa Barbara, CA, USA). Vimentin filaments were attached to a freshly cleaved mica surface by physisorption. Cantilevers (MLCT, Bruker, Billerica, MA, USA) were calibrated using the thermal noise method; spring constants were found to be 50 to $60 \mathrm{pN} / \mathrm{nm}$. Stretching was performed by catching the freely fluctuating end of only partly attached vimentin filaments with the cantilever via physisorption and repeatedly displacing the cantilever from the mica surface with a loading rate of $5 \mu \mathrm{m} / \mathrm{s}$. A final force of about $8 \mathrm{nN}$ was reached without breaking the filaments.

The raw data (force-distance curves) are processed by selfwritten MATLAB code to obtain force-strain curves for every filament. The strain is defined as $\epsilon=\Delta L / L$, where $\Delta L$ is the difference between the length of the extended filament and the length of the filament $L$. The unstretched length $L$ is measured at the largest measured force before reaching $5 \mathrm{pN}$. The individual experimental data sets are shown in Fig. 2 (thin lines), where each color represents one loading rate, altogether covering 2 orders of magnitude. The averages of the measured curves are calculated using a MATLAB TOOLBOX written by Carlos J. Dias applying the theory described in Refs. [23,24], and are represented by the thick colored lines.

In the individual OT data sets, three different regimes can be discerned. Comparison of our data to literature suggests that the steep linear increase at low strain represents the

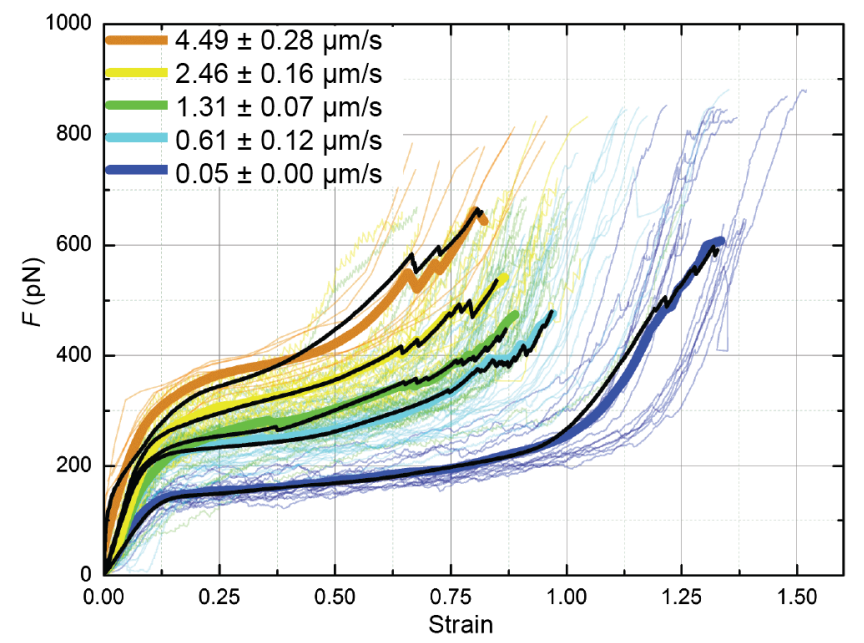

FIG. 2. Force-strain curves recorded with the OT at different loading rates (color code, see legend) of individual vimentin filaments (thin lines) and corresponding average (thick line). The black lines show the averaged fits for each loading rate. 
elastic stretching of the $\alpha$-helical domains [16,25,26], whereas during the second plateaulike regime, starting at a strain of about 0.1 , the $\alpha$ helices are uncoiled and $\beta$ sheets are formed instead $[16,25,27]$. In the third regime a clear stiffening with respect to the plateau region can be observed which, according to simulations [16], might be due to increased pulling on the $\beta$ sheets. Previously, it has been shown that cells are indeed able to resist strains in the same order of magnitude as reached here [8].

Furthermore, we observe a clear loading rate dependence of the force-strain curves. With increasing loading rate the force value of the plateau region increases and strain range of the plateau regime decreases. Whereas the strain at which the plateau is reached does not change much between the loading rates, the strain at which the stiffening starts decreases with increasing loading rate.

To describe the force-extension curves of vimentin filaments in the strain range accessible by OT we adopt an elastically coupled two-level model introduced by Rief et al. [28]. In brief, the filament is modeled as a system of entropic springs coupled to an elastic element [Fig. 3(a)]. Each monomer can assume different states with distinct lengths and energies, in case of vimentin a shorter $\alpha$-helical ground state of length $l_{\alpha}$ and an extended $\beta$ sheet of length $l_{\beta}$. It is known that the vimentin monomer consists of three $\alpha$ helices, which are assumed to react independently to the applied force and are indicated by subscripts $(i=1,2,3$ corresponding to the $1 \mathrm{~A}, 1 \mathrm{~B}$, and 2 domain) in the following. The $\alpha$ helices switch stochastically into a $\beta$-sheet conformation following force-dependent Bell-Evans kinetics.
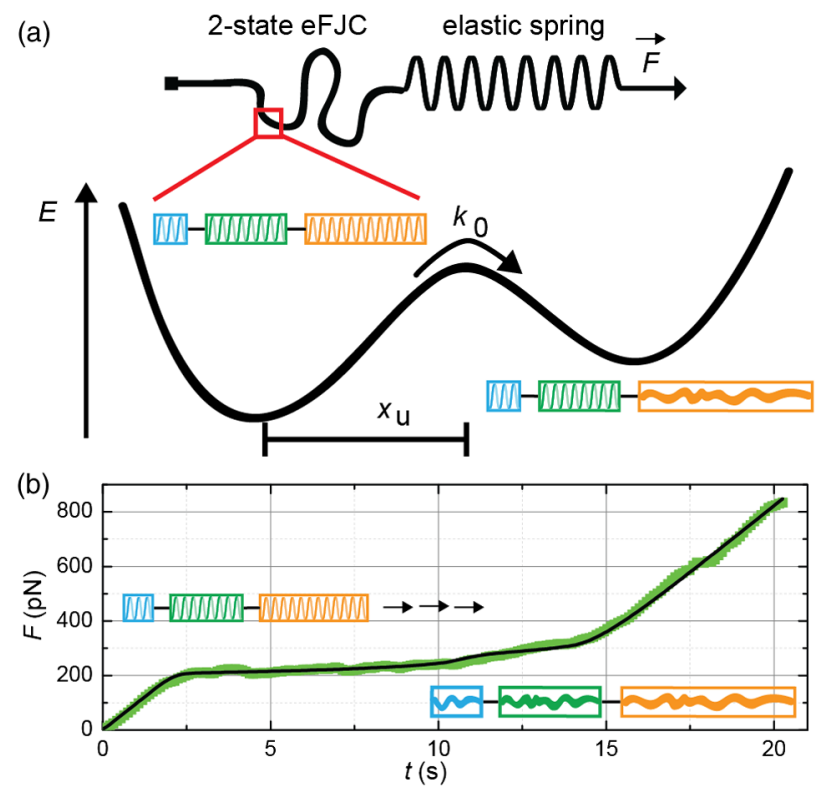

FIG. 3. (a) Sketch of the mechanical model consisting of a twostate eFJC and an elastic spring (top). A double-well potential is assumed to describe the unfolding reaction of the $\alpha$-helical subunits of a vimentin monomer (bottom). (b) Equation (1) fitted to a typical force-time curve of a single vimentin filament stretched at a loading rate of $0.6 \mu \mathrm{m} / \mathrm{s}$.
This approach allows us to obtain the number $N_{\beta, i}$ of monomers with $\beta$-sheet conformation $\left(d N_{\beta, i} / d t\right)=$ $\left(N_{0}-N_{\beta, i}\right) k_{0, i} \exp \left[F x_{u, i} /\left(k_{B} T\right)\right]$, where the $i$ th subunit is in the extended state. Here, the total number of monomers arranged in series is $N_{0}=N_{\alpha, i}+N_{\beta, i}$, the zero-force transition rate of the respective three subunits is $k_{0, i}$, and their potential width is $x_{u, i}$. In the vimentin filament, on average 32 monomers are arranged in parallel. We assume that parallel monomers are captured by this model by decreasing the potential width $x_{u}$ as described by Friddle et al. [29]. $x_{u, i}$ is, therefore, an apparent value dependent on the number of parallel monomers. The contour length of the filament is $L_{\mathrm{c}}=N_{0} l_{0}+\sum_{i}\left(N_{\beta, i} l_{\beta, i}+N_{\alpha, i} l_{\alpha, i}\right)$, where $l_{0}$ is the combined length of the linker regions. We use the equivalent Freely Jointed Chain (eFJC) model with the Kuhn length $L_{K}$, which gives a good description in the force range observed experimentally [30], to model the entropic behavior of the filament under stress (see Supplemental Material [22] for a more detailed description of the model). In combination with an effective spring constant $k_{\text {eff }}$, which includes the elastic behavior of the filament as well as the spring constant of the AFM cantilever or the OT, we obtain an expression for the time-dependent force $F$ for a linear extension of the filament with constant loading rate $v$ :

$$
F(t)=k_{\text {eff }}\left(v t-L_{c}(F, t)\left(1-\frac{F_{K}}{F}\right)\right)
$$

Equation (1) is solved analytically for $F$ using the numerical solution for the rate equation and fitted to the data. The model describes the experimental data remarkably well [see Fig. 3(b) for an example fit to an OT data curve]. To reduce the number of fit parameters, $l_{\alpha, i}$ and $l_{\beta, i}$ are estimated from structural data [5-7]: $l_{\alpha, 1}=5.4, l_{\alpha, 2}=13.8, l_{\alpha, 3}=20.9$, $l_{\beta, 1}=12.6, l_{\beta, 2}=32.2, l_{\beta, 3}=48.7$, and $l_{0}=29.7 \mathrm{~nm} . k_{0, i}$ is set to $k_{0, i}=3.3 \times 10^{-5} \mathrm{~s}^{-1}$, as found by Rief et al. for the unfolding of $\alpha$-helical spectrin repeats [31].

All experimental data curves are fitted individually with independent starting parameters. We find multiple local minima, i.e., sets of parameters able to describe all different experimental curves with little variance (see Supplemental Material [22]). The parameter set with the lowest variance is $\left(\right.$ mean $\pm \mathrm{SD}$ ) $\bar{x}_{u, 1}=0.09 \pm 0.04, \quad \bar{x}_{u, 2}=0.12 \pm 0.04$, and $\bar{x}_{\mathrm{u}, 3}=0.18 \pm 0.06 \mathrm{~nm}$. The effective spring constant of the filament was found to be $\bar{k}_{\text {eff }}=0.22 \pm 0.06 \mathrm{pN} / \mathrm{nm}$, corresponding to a Young's modulus $E=k_{\text {eff }}\left(L / \pi r^{2}\right) \approx$ $27 \pm 10 \mathrm{MPa}$, and a persistence length of $L_{P}=E \pi r^{4} /$ $\left(4 k_{B} T\right) \approx 3.3 \mu \mathrm{m}$. Here, we include that the spring constants of the OTs and the AFM $(1.23$ and $50 \mathrm{pN} / \mathrm{nm}$, respectively) are large compared to $k_{\text {eff }}$. Given that the radius $r \approx 10 \mathrm{~nm}$ of the filament enters the persistence length with a power of 4 , this result is in very good agreement with previous measurements of $L_{P}[11,12]$. The average of all individual fits for each loading rate in the same strain range as the curves of the averaged experimental data sets is shown as black lines in Fig. 2. 
The OT setup provides superior control over the experiment due to the simultaneous imaging modality and the microfluidic chip. However, the maximum forces reached are on the order of $800 \mathrm{pN}$, and Fig. 2 clearly shows that the maximum strain is not yet reached at these forces. Previous AFM experiments have shown that rupture of the filaments occurs at a strain of about 2.6 [13], whereas the maximum strain reached in our OT experiments is 1.5. Therefore, we compare force-strain curves obtained by OT with AFM data, as shown in Fig. 4. The open circles represent the average curve of all AFM measurements, including the standard deviations as error bars in direct comparison to the OT data (solid symbols) at a similar loading rate. In the AFM experiment the filament is stretched between the AFM tip and a mica surface, providing less control over the orientation of the filament compared to a filament trapped between two beads. Therefore, the variance of force between the individual filaments is higher than for OT data. However, the average curves of both experiments overlap remarkably well in the lower force regime, confirming that we measure the response of individual filaments in the AFM experiments. Additionally, the AFM measurement allows us to apply forces up to $8 \mathrm{nN}$ and, therefore, to reach a strain of up to 3.5 , albeit, still without breaking the filaments. For lower strains AFM force-strain curves replicate the three stress regimes found in the OT experiments. At larger strains, however, two additional regimes are observed: a second flat region with only little change in force at strains between 1.25 and 2, and a third linear regime at strains greater than 2 is found. A second force plateau has been predicted by molecular dynamics simulations, where it was assigned to sliding between dimers within a tetramer [16].

In combination, OT and AFM measurements provide a precise and quantitative approach to the force-strain relationship for individual vimentin IFs. The data can be well

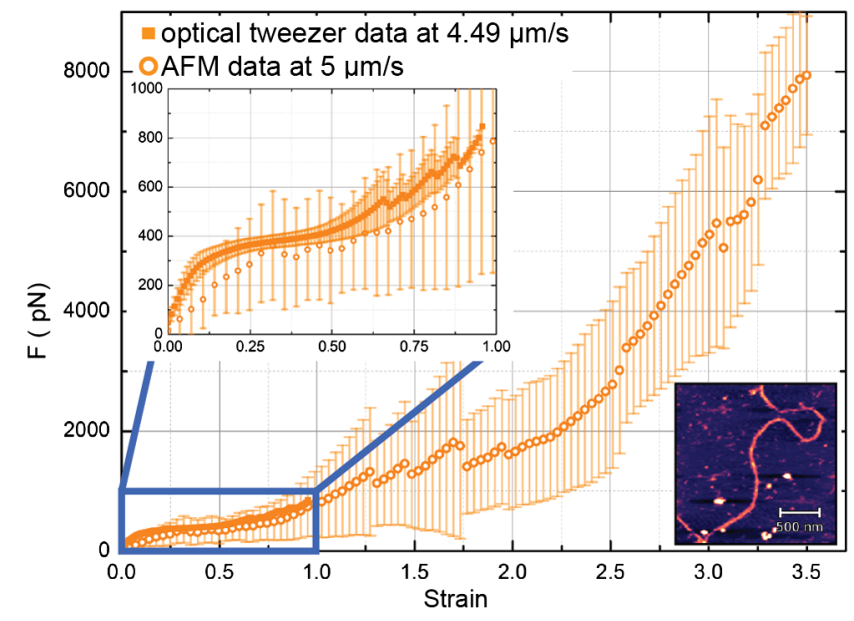

FIG. 4. Comparison of AFM (open circles) and OT (solid symbols) experiments using approximately the same loading rate. The top left inset shows an enlargement of the part where the data of OT and AFM measurements overlay. The bottom right inset shows an AFM image of vimentin filaments on a mica surface. described up to a strain of 1.2 by an elastically coupled system of multiple two-state elements supporting the hypothesis that subsequent stochastically independent unfolding of $\alpha$ helices into more extended $\beta$-sheet structures is responsible for the first force plateau and is thereby contributing to the remarkable extensibility of these filaments. When a critical force defined by $k_{0}$ and $x_{u}$ is reached [32], the filament extends very rapidly leading to a force plateau as observed in the experiment. It is necessary to assume an independent reaction of the three $\alpha$-helical domains in the rod to capture the change in plateau length between force curves at different loading rates while keeping $l_{\beta, i}$ fixed at the values known from structural data. The preferred order in which the three subunits unfold is governed by the potential width $x_{u, i}$ : The subunit with the largest potential width shows the strongest coupling to the applied force and has therefore the highest probability to unfold first. Interestingly, our fits indicate a high preference that coil 2 (corresponding to $i=3$ ) opens up first. The reason could be the so-called stutter region which is found precisely six heptads spaced from the $C$-terminal end of coil 2 in all IF types [33,34]. At this position the heptad repeat, characteristic of coiled coils, is disrupted, either due to three missing or four extra amino acids $[33,35]$. Notably, this defect does not compromise the coiled-coil geometry, but leads to an almost parallel run of the two $\alpha$ helices $[34,36]$ and, therefore, changes the angle between the direction of the applied force and the direction of the bonds that break during the stretching process [26]. Thus, the stutter region is less stable than an intact coiled coil and might be the reason why coil 2 responds most sensitively to applied forces. From a biological point of view this is in agreement with the hypothesis that IFs act as a "safety belt" for cells [16]; that is, they are easily deformable and thus mechanically almost not detectable at slow deformation but stiff and therefore preserving the cell shape at fast deformation. This aspect is reflected in the shortened plateau regime (see Fig. 2) at higher loading rates. At slow deformation, the filaments can be extended to about twofold their original length (strain of 1) at a comparatively low force. When pulled on faster, however, already at a strain of 0.5 , increased forces are necessary for further extension. Our numerical model implies a simple mechanism for this observation based on the independent unfolding of the three individual coils forming the monomer. Forces as strong as applied by AFM here occur rarely in nature; therefore, the second nonlinear regime may be interpreted as a controlled failure mechanism, allowing the filament to withstand extreme forces and large deformation.

Our data agree well with previous molecular dynamics simulations [16], not only with regard to the different observed regimes in the force-strain curve, but even concerning the numerical values for strains, even though Qin et al. only modeled stretching of dimers and tetramers. Within a fully assembled vimentin filament, about 32 monomers, albeit with some variation to this number 
[37], are arranged in parallel. In our model we assume that both monomers in series and in parallel arrangement react independently. It is, however, expected that the monomers elongate in a coordinated way. Future experiments involving more sophisticated imaging in parallel to the mechanical stretching may help to approach these aspects.

In conclusion, our experimental results support the hypothesis that IFs provide strength to cells under large deformation and that unfolding enables them to absorb large amounts of energy $[34,38]$. During a slow deformation, vimentin acts as a very soft material, while a faster deformation shortens the plateau region and leads to a "stiffening" of the filament. More generally, the force-dependent switching between $\alpha$ helices to $\beta$ sheets as well as breaking of lateral bonds between individual subunits can be understood in the light of molecular bond-breaking taking place on different levels of hierarchy in soft biological matter, as recently, shown by Kurniawan for the blood protein fibrin [39,40]. Thus, we believe that our study of individual cytoskeletal IFs will help to further quantify the underlying physical phenomena of soft matter mechanics.

We are grateful for fruitful discussions and technical support by J. Cabanas Danes, H. Herrmann, F. C. MacKintosh, and K. Kroy. The work was financially supported by the Deutsche Forschungsgemeinschaft (DFG) in the framework of SFB755 (project B7), SFB937 (project A17), and the GGNB (Grant No. GSC 226/2). The research leading to these results has received funding from Laserlab-Europe (Grant Agreement No. 654148, European Union's Horizon 2020 research and innovation programme).

*sarah.koester@phys.uni-goettingen.de

[1] F. Huber, A. Boire, M. P. López, and G. H. Koenderink, Curr. Opin. Cell Biol. 32, 39 (2015).

[2] J. Block, V. Schroeder, P. Pawelzyk, N. Willenbacher, and S. Köster, Biochim. Biophys. Acta 1853, 3053 (2015).

[3] K. Seltmann, A. W. Fritsch, J. A. Käs, and T. M. Magin, Proc. Natl. Acad. Sci. U.S.A. 110, 18507 (2013).

[4] L. Ramms, G. Fabris, R. Windoffer, N. Schwarz, R. Springer, C. Zhou, J. Lazar, S. Stiefel, N. Hersch, U. Schnakenberg et al., Proc. Natl. Acad. Sci. U.S.A. 110, 18513 (2013).

[5] S. Nicolet, H. Herrmann, U. Aebi, and S. V. Strelkov, J. Struct. Biol. 170, 369 (2010).

[6] A. A. Chernyatina, D. Guzenko, and S. V. Strelkov, Curr. Opin. Cell Biol. 32, 65 (2015).

[7] UniProt, http://www.uniprot.org/uniprot/P08670 p. viewed: 2016.08.05 (2016).

[8] L. Kreplak and D. Fudge, BioEssays 29, 26 (2007).

[9] E. E. Charrier and P. A. Janmey, Methods Enzymol. 568, 35 (2016).

[10] S. Köster, D. A. Weitz, R. D. Goldman, U. Aebi, and H. Herrmann, Curr. Opin. Cell Biol. 32, 82 (2015).

[11] B. Nöding and S. Köster, Phys. Rev. Lett. 108, 088101 (2012).
[12] N. Mücke, L. Kreplak, R. Kirmse, T. Wedig, H. Herrmann, U. Aebi, and J. Langowski, J. Mol. Biol. 335, 1241 (2004).

[13] L. Kreplak, H. Bär, J. F. Leterrier, H. Herrmann, and U. Aebi, J. Mol. Biol. 354, 569 (2005).

[14] L. Kreplak, H. Herrmann, and U. Aebi, Biophys. J. 94, 2790 (2008)

[15] C. Guzman, S. Jeney, L. Kreplak, S. Kasas, A. J. Kulik, U. Aebi, and L. Forro, J. Mol. Biol. 360, 623 (2006).

[16] Z. Qin, L. Kreplak, and M. J. Buehler, PLoS One 4, e7294 (2009).

[17] H. Herrmann, L. Kreplak, and U. Aebi, Methods Cell Biol. 78, 3 (2004).

[18] S. Winheim, A. R. Hieb, M. Silbermann, E.-M. Surmann, T. Wedig, H. Herrmann, J. Langowski, and N. Mucke, PLoS One 6, e19202 (2011).

[19] N. Mücke, T. Wedig, A. Bürer, L. N. Marekov, P. M. Steinert, J. Langowski, U. Aebi, and H. Herrmann, J. Mol. Biol. 340, 97 (2004).

[20] A. Candelli, G. J. L. Wuite, and E. J. G. Peterman, Phys. Chem. Chem. Phys. 13, 7263 (2011).

[21] I. Heller, G. Sitters, O. D. Broekmans, G. Farge, C. Menges, W. Wende, S. W. Hell, E. J. G. Peterman, and G. J. L. Wuite, Nat. Methods 10, 910 (2013).

[22] See Supplemental Material at http://link.aps.org/ supplemental/10.1103/PhysRevLett.118.048101 for a movie of the filament pulling, a more detailed description of the theoretical model, a comparison of the worm-like chain and freely jointed chain models and the fit results.

[23] A. Savitzky and M. J. E. Golay, Anal. Chem. 36, 1627 (1964).

[24] P. Barak, Anal. Chem. 67, 2758 (1995).

[25] Z. Qin and M. J. Buehler, J. Mol. Model. 17, 37 (2011).

[26] T. Ackbarow and M. J. Buehler, Exp. Mech. 49, 79 (2009).

[27] E. G. Bendit, Textile research Journal: publication of Textile Research Institute, Inc and the Textile Foundation 30, 547 (1960).

[28] M. Rief, J. M. Fernandez, and H. E. Gaub, Phys. Rev. Lett. 81, 4764 (1998).

[29] R. W. Friddle, A. Noy, and J. J. de Yoreo, Proc. Natl. Acad. Sci. U.S.A. 109, 13573 (2012).

[30] N. M. Toan and D. Thirumalai, Macromolecules 43, 4394 (2010).

[31] M. Rief, J. Pascual, M. Saraste, and H. E. Gaub, J. Mol. Biol. 286, 553 (1999).

[32] G. Bell, Science 200, 618 (1978).

[33] H. Herrmann and U. Aebi, Annu. Rev. Biochem. 73, 749 (2004).

[34] T. Ackbarow and M. J. Buehler, J. Mater. Sci. 42, 8771 (2007).

[35] S. V. Strelkov, H. Herrmann, N. Geisler, T. Wedig, R. Zimbelmann, U. Aebi, and P. Burkhard, EMBO J. 21, 1255 (2002)

[36] A. A. Chernyatina, S. Nicolet, U. Aebi, H. Herrmann, and S. V. Strelkov, Proc. Natl. Acad. Sci. U.S.A. 109, 13620 (2012).

[37] H. Herrmann, M. Haner, M. Brettel, S. A. Muller, K. N. Goldie, B. Fedtke, A. Lustig, W. W. Franke, and U. Aebi, J. Mol. Biol. 264, 933 (1996).

[38] D. S. Fudge, K. H. Gardner, V. T. Forsyth, C. Riekel, and J. M. Gosline, Biophys. J. 85, 2015 (2003).

[39] N. A. Kurniawan, B. E. Vos, A. Biebricher, G. J. L. Wuite, E. J. G. Peterman, and G. H. Koenderink, Biophys. J. 111, 1026 (2016).

[40] K. Kroy, Biophys. J. 111, 898 (2016). 